\title{
REVIEW
}

\section{Patterns of Reactions to Red Pigment Tattoo and Treatment Methods}

Emily Forbat $\cdot$ Firas Al-Niaimi

Received: December 21, 2015 / Published online: March 14, 2016

(C) The Author(s) 2016. This article is published with open access at Springerlink.com

\section{ABSTRACT}

Tattoos are common and used extensively as either body art or cosmetic make-up; more rarely, they can be traumatic in nature. We have systemically analysed the literature for the patterns of red pigment tattoo reactions and their treatment options. Our search identified 18 articles; there was 1 non-randomised controlled trial, and the rest were small case studies. In total 139 patients were included within the studies. This review systematically analyses the different subsets of red tattoo reactions including lichenoid, dermatitis, granulomatous, pseudolymphomatous and miscellaneous reactions. The current evidence for the treatment for the above is presented. Dermatitis and lichenoid reactions appear to be

Enhanced content To view enhanced content for this article go to http://www.medengine.com/Redeem/ 1944F0605D19D438.

E. Forbat

King Edward VII Hospital, 5-10 Beaumont St, London W1G 6AA, UK

F. Al-Niaimi $(\varangle)$

Dermatological Surgery and Laser Unit, St John's Institute of Dermatology, St Thomas' Hospital, Westminster Bridge Road, London SE1 7EH, UK e-mail: firas55@hotmail.com the most common subtype of red pigment reactions with various treatment methods applied showing laser intervention to have some degree of success.

Keywords: Dermatitis; Granulomatous; Lichenoid; Pseudolymphomatous; Tattoos

\section{INTRODUCTION}

Tattoos are very popular in society today with their prevalence varying depending on the age group, ethnicity and location demographics, with the range thought to be between 5\% to $40 \%$ in adults [1]. Tattoos can be classified as traumatic, cosmetic or decorative and their placement can be professional or amateur [2].

Complications of tattoos can be divided into cutaneous or systemic and can have an impact on the quality of life [3]. Cutaneous complications can occur either immediately or be delayed. Although there is no universally accepted classification, the complications are often classified according to the clinical and histological features with some overlap [4]. Examples of delayed reactions include allergic 
contact dermatitis, granulomatous dermatitis, lichenoid dermatitis and pseudolymphomatous reactions $[4,5]$.

In this article we discuss the different types of reactions to red tattoo pigment with a review of the literature with regards to the treatment for each pattern.

\section{METHODS}

A literature search was performed in November 2015 to review the current literature on red tattoo reactions in terms of classification and their treatments. A PubMed and Google scholar search was carried out with the search criteria "red tattoo", "reaction", "allergic" and "treatment". Articles were selected depending on their relevance involving red tattoo reactions only. This article is based on previously conducted studies and does not involve any new studies of human or animal subjects performed by any of the authors.

\section{TYPES OF RED TATTOO PIGMENT (ORGANIC VERSUS INORGANIC)}

Red tattoo pigment can be either organic or inorganic. Inorganic red pigment includes mercury, cadmium selenide and sienna (ferric hydrate) [6]. Organic red pigment includes sandalwood and brazilwood (or Caesalpinia echinata), both organic vegetable dyes [7, 8].

The red pigment can also be made with cinnabar (a mercury derivative) and it is this that is thought to cause the cell-mediated delayed hypersensitivity reaction [9]. Mercury in red tattoos has also been reported to cause lichenoid reactions [10] and rarely massive pseudoepitheliomatous hyperplasia [11].

Red tattoo pigments are thought to contain toxic metals, which predispose the skin to a higher incidence of adverse reactions, particularly lichenoid and allergic contact dermatitis [8, 12, 13]. Swoden et al. studied the chemical composition of red pigment tattoos in 18 patients who developed cutaneous reactions and found aluminium, iron, calcium, titanium, silicon, mercury and cadmium within the pigment, all of which could trigger cutaneous inflammation [8].

One study tattooed pig and human skin with the pigment red 22 (commonly used and thought to be hazardous). The authors then extracted the pigment to assess the concentration in the skin and found high concentrations of the pigment (mean $2.53 \mathrm{mg} /$ $\mathrm{cm}$ [3]), supporting the frequent incidence of complications from modern tattoos, particularly those involving red pigment [14].

Another study reviewed the histological pattern of skin biopsies from 19 patients who had red tattoo reactions. The majority (78\%) of samples demonstrated dermatitis with evidence of T-lymphocytes and Langerhans cells, which further supports the presence of an allergic phenomenon [15].

\section{PATTERNS OF RED TATTOO PIGMENT REACTIONS}

\section{Dermatitis}

Eczematous reactions to red tattoos-alongside lichenoid-are the most common type of reaction observed, being either allergic contact dermatitis or photo-allergic dermatitis [16]. Photo-allergic reactions most commonly occur secondary to the cadmium subset of red pigment [17] and skin protection from ultraviolet light has been shown to exert protective effects [18]. 
One study in Denmark found that approximately half of sunbathers with red or black tattoos in particular suffered from photo-allergic reactions. They hypothesised that reactive oxygen species may play a role in triggering a dermatitis-type reaction in such individuals [19].

One interesting study carried out patch testing in 90 patients with tattoo reactions over a 4-year period. They found that red tattoo reactions did not predispose patients to have a positive patch test result with the common tested allergens. They hypothesised that the allergic result is due to haptenisation, possibly due to 'photochemical cleavage of red azo pigment' [20]. To strengthen the argument that patch testing does not correlate with red tattoo reactions, Anthony and Harland also found patch testing carried out prior to laser removal was negative to mercury at 48 and $96 \mathrm{~h}$ [21].

The exact understanding of the mechanisms behind the eczematous reactions observed in red tattoos is still lacking with type I-III hypersensitivity reactions playing a role [9]; however there is no conclusive evidence to date.

\section{Lichenoid}

In 1978 it was first hypothesised that tattoos may imitate a localised antigenic challenge, which in turn can cause a lichenoid pattern of reaction [22]. Lichenoid reactions in red organic tattoos have been shown to elicit a cytotoxic inflammatory response of the tissue [12] with lichenoid basal damage [23] thought to be produced by a delayed cellular hypersensitivity to metal particles [24].

The current consensus for the most common tattoo reaction is conflicting, with some papers stating dermatitis is the most common type, and others lichenoid [25]. Tattoo lichenoid reactions are most commonly associated with red pigment, particularly because of mercury [26].

\section{Pseudolymphomatous}

Pseudolymphoma is a term given to a histological entity and has been reported to occur as a complication of tattoos that is histologically indistinct from malignant $\mathrm{T}$ - or B-cell lymphoma [4]; however the lymphoproliferative process is benign [27]. The clinical presentation is a pruritic plaque within the tattoo, often initially mistaken for lymphoma. The distinguishing factor between pseudolymphoma and lymphoma is the polyclonal nature of the lymphocytes [4]. Amann et al. propose it can only be distinguished from lymphoma with the aid of electron microscopy, immunohistochemistry and molecular biology [28].

In one reported case a 35-year-old male reacted with infiltrated nodules within the red areas of his tattoo 2 months post tattooing [29], whilst another paper reported a pseudolymphomatous allergic immune reaction 6 months post initial tattooing [30].

\section{Granulomatous}

Similar to lichenoid reactions, granulomatous reactions are thought to occur as a result of delayed hypersensitivity reactions to the presence of the red pigment [31].

One case study found a granulomatous reaction in the red pigment of four multi-coloured tattoos; interestingly only one of these lesions contained the metal mercurywhich is thought to commonly cause these reactions in red tattoos [7].

Allopurinol has proved to be useful in the treatment of red tattoo granulomas, with 
improvement of symptoms during a 6-month course and regression of results upon cessation of treatment [32].

\section{Miscellaneous Reaction Patterns}

Although dermatitis and lichenoid reactions are by far the most common, there have been reports of some rarer forms of reactions to red tattoo pigment. Litvinov and Sassevile reported a case of pyoderma gangrenosum [33] in a red tattoo consistent with the Koebner phenomenon. One group reported a red tattoo granulomatous dermatitis that was histologically similar to granuloma annulare presented post acquisition of a red pigment tattoo [31].

A 2003 paper found that the azo pigments and quinacridones found in red tattoo pigment led to skin reactions with differing histology found on biopsy: some lichenoid, some eczematous and others pseudolymphomatous [34] (Table 1).

There have also been several cases of systemic sarcoidosis presenting initially as a granulomatous tattoo reaction [35-38]. One study looked at sarcoid development within cosmetic tattooing of the eyebrows and lips and observed that a granulomatous reaction containing a foreign body should not anticipate an exclusion of systemic sarcoidosis. A sarcoid within a tattoo is an example of the Koebner phenomenon [39]. Whether a sarcoid is a reaction to the tattoo itself or coincidental with a systemic disease remains a conundrum. It is suggested that in case of a tattoo sarcoid a search for systemic sarcoidosis is warranted [35].

Finally there are reports of deep-seated infections manifesting within tattoos, such as leprosy, syphilis, tuberculosis and Mycobacterium chelonae [40, 41].

\section{TREATMENT OF RED PIGMENT REACTIONS}

Multiple treatment options for red tattoo pigment reactions have been employed with little background evidence for their use. Medical treatment options have included allopurinol use as well as topical and intralesional corticosteroids [21] and secondary measures such as sun protection and antibiotics $[18,32$, 42, 43]. Effective laser treatment has been demonstrated with both Q-switched Nd:YAG and erbium:YAG lasers [21, 44]. Anthony and Harland demonstrated successful laser

Table 1 Clinico-pathological patterns of red tattoo reactions

\begin{tabular}{ll}
\hline Skin reaction & Histology \\
\hline Allergic contact dermatitis & Acanthosis, spongiosis, perivascular lymphocytic inflammatory infiltrate \\
Lichenoid dermatoses & Acanthosis and thickening of the stratum corneum \\
Granulomatous & Giant cell positive or negative \\
Pseudolmphomatous & T- or B-cell lymphoma equivalent \\
Pseudoepitheliomatous hyperplasia & Reactive changes: similar to SCC and keratoacanthoma \\
& Irregular acanthosis \\
& Low mitotic activity \\
\hline
\end{tabular}


treatment in seven patients within an open non-randomised clinical trial [21] and De Argila presented a successful outcome of one case of a lichenoid tattoo reaction treated with five treatment sessions of erbium:YAG [44].

A carbon dioxide $\left(\mathrm{CO}_{2}\right)$ laser has also been used. Kyanko et al. treated two cases of red tattoo dermatitis with a $\mathrm{CO}_{2}$ laser in cases previously resistant to topical and intralesional corticosteroids [45]. Madan found that the $\mathrm{CO}_{2}$ laser was particularly useful for red ink tattoo granulomas that were recalcitrant to conventional steroid treatment [46]. Of note, the $\mathrm{CO}_{2}$ laser has also been reported to trigger the generalisation of localised tattoo dermatitis [47].

Finally, there is the option of surgical excision. The appropriateness of this will of course depend on the extent of the reaction and the size of the tattoo. Eczematous reactions have been successfully treated with excision and concomitant low-dose intralesional corticosteroids [43].

\section{CONVENTIONAL METHODS OF TATTOO REMOVAL}

Tattoo removal dates back to the Roman era when dried Spanish flies were used-the cantharides induced skin irritation and blistering [48]. Today nano- and picosecond lasers are the gold standard for removing tattoos of all types: professional, cosmetic or even traumatic [49]. Targeted photothermolysis is believed to create acoustic pressure leading to pigment fragmentation into the surrounding tissues and to enable it to be engulfed by macrophages leading to the subsequent removal of the pigment from the tattoo [22]. Although the treatment is largely safe, depigmentation and occasionally scarring are potential long-term complications [50].

There have been reports of Q-switched lasers (nano-second) causing allergic reactions following their use in tattoo removal. This is thought to be due to the dispersion of the pigment triggering an immune response $[9,16]$. Paradoxically, it is the Q-switched double-frequency Nd:YAG laser that is most beneficial for removing red pigment within tattoos. The current thinking of Q-switched laser treatment triggering an anaphylactic reaction has been challenged and dismissed by some [21].

\section{RESULTS OF THE LITERATURE SEARCH}

Our search identified 18 articles, with the majority being case studies. There was one open non-randomised controlled trial on lichenoid tattoo reactions, and the remainder were all case studies (Table 2). In total 139 patients were included within the studies. The red tattoo reactions described and treated included dermatitis (four case studies, overall $n=23$ ), lichenoid (four case studies, one open non-randomised control trial, overall $n=11$ ), granulomatous (three case studies, overall $n=6$ ) and pseudolymphomas (two case studies, overall $n=2$ ). Miscellaneous studies included a single case of pyoderma gangrenosum $(n=1)$, a case study on pigment darkening post Q-switched and pulsed laser treatment [including Q-switched Ruby, Q switched Nd:YAG and pulsed green dye (510 nm lasers) $(n=5)]$, a case of successful $\mathrm{CO}_{2}$ laser removal of a facial red tattoo $(n=1)$ and a large study $(n=90)$ reviewing patch testing outcomes in patients with red tattoo reactions. 


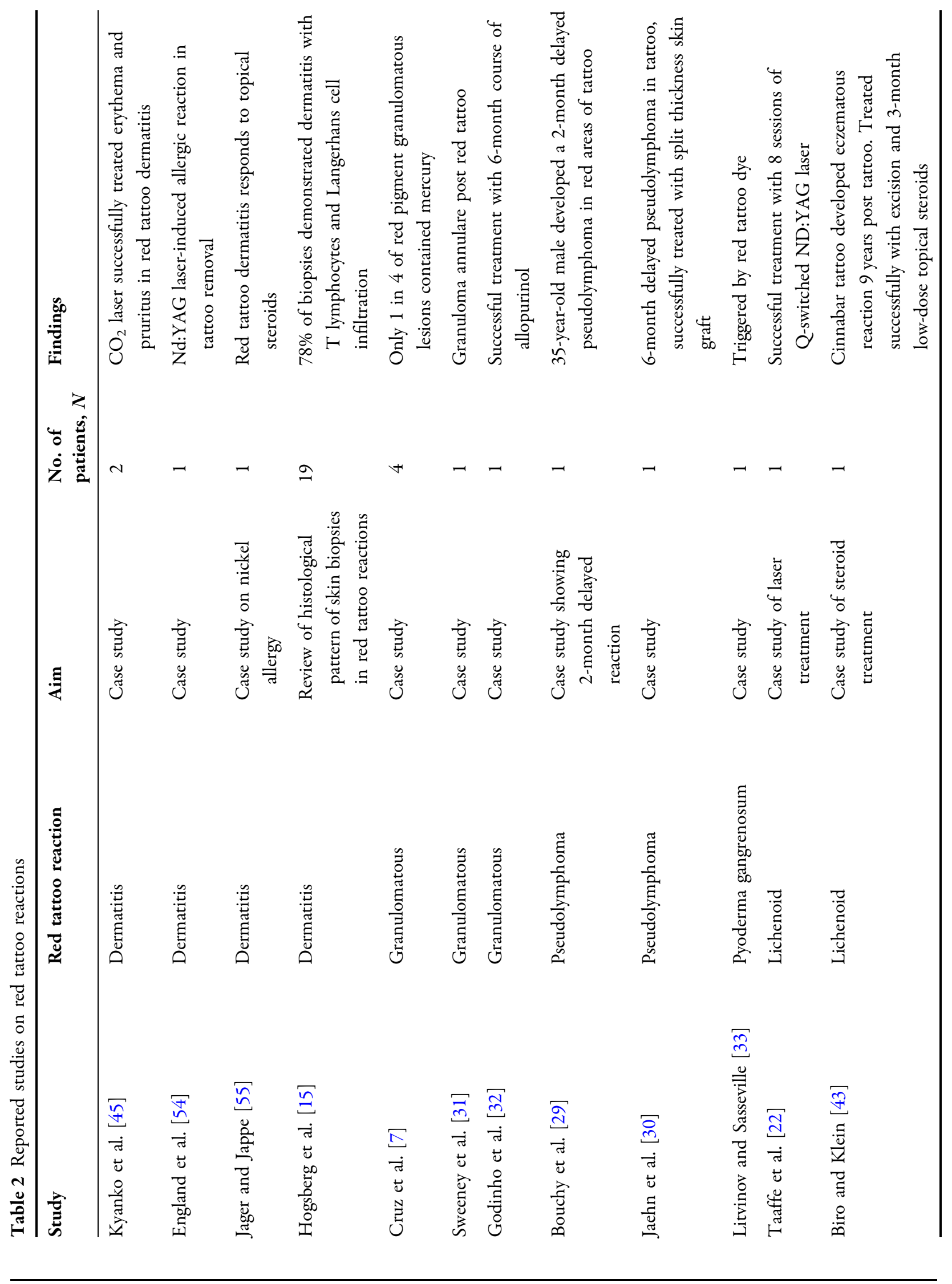




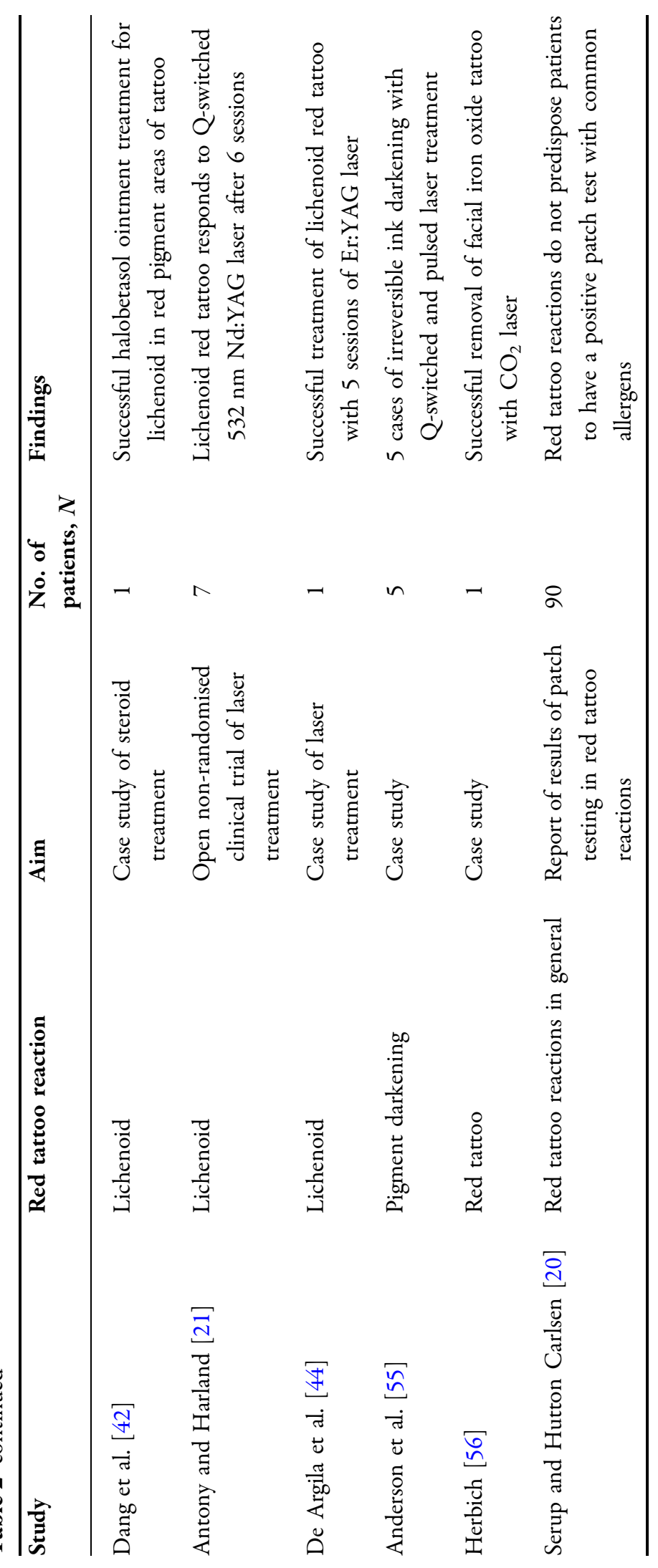


Of the studies that reviewed treatment outcomes in red tattoo reactions, two were treated with topical steroids, two with $\mathrm{CO}_{2}$ lasers, four with Q-switched Nd:YAG, one with Er:YAG, one with allopurinol, one with a split-thickness skin graft and one with surgical excision (Table 2).

\section{DISCUSSION}

Tattoos are popular and it is likely that reactions to tattoo pigment will continue to develop in various forms. Red pigment is the most common cause of reactions in tattoos and this can present in various clinical and histological variants with dermatitis and lichenoid being the most common. The literature on tattoo reactions, their classifications and treatments is not exhaustive. The high incidence of reactions in red tattoos is attributed to the toxic metals often found within the pigment, which predispose the skin to a higher incidence of adverse reactions $[8,12,13]$. Furthermore, there is no quality control or legislation regarding the inks contained within red tattoos [51], especially inorganic cinnabar tattoos, highlighting that a safer outcome may be found with synthetic tattoos whereby the dye within them is [21]. It has been reported that some inks have been obtained from the clothing industry (red dyes for clothes) and that there may have been batches contaminated in some way. One study analysed the decomposition of tattoo pigments using liquid chromatography and mass spectrometry; they found lasers broke down the pigments to produce 2-methyl-5-nitroaniline, 2-5-dichloraniline and 4-nitro-toluene. These materials are not only toxic but postulated to be carcinogenic $[52,53]$, which may contribute to the high incidence of red tattoo reactions.

Most interestingly, the hypothesis that laser treatment for tattoo reactions can lead to anaphylaxis [9] has been challenged with successful improvement of lichenoid tattoo reactions in seven patients within one case study [21]. There have been promising results with both Q-switched Nd:YAG lasers and erbium:YAG [21, 22, 44] as well as successful outcomes with $\mathrm{CO}_{2}$ lasers [45]. One could speculate that a type- 1 anaphylactic reaction is unlikely to occur with the type 3 and 4 reactions demonstrated with red tattoo pigments. Another important point for clinicians to recognise is that patch testing does not correspond to the tattoo reaction outcome. All of the patch testing carried out by Anthony and Harland was negative, yet the patients had red tattoo reactions [21], suggesting that this step can be omitted with regards to clinical work-up. The evidence for this entire literature search, although enlightening, is based only on small case studies and therefore larger studies are required to cement these encouraging outcomes.

\section{CONCLUSION}

This review systematically analyses the different subsets of red tattoo reactions including lichenoid, dermatitis, granulomatous, pseudolymphomatous and miscellaneous reactions. Dermatitis and lichenoid reactions appear to be the most common subtype of red pigment reactions, with the various treatment methods applied showing laser intervention in fact to have positive outcomes, contrary to the hypothesis of anaphylaxis risk when it is used to treat red tattoo reactions. 


\section{ACKNOWLEDGMENTS}

No funding or sponsorship was received for this study or publication of this article.

All named authors meet the International Committee of Medical Journal Editors (ICMJE) criteria for authorship for this manuscript, take responsibility for the integrity of the work as a whole and have given final approval for the version to be published.

Disclosures. Dr. Emily Forbat and Dr. Firas Al-Niaimi have no disclosures.

Compliance with Ethics Guidelines. This article is based on previously conducted studies and does not involve any new studies of human or animal subjects performed by any of the authors.

Open Access. This article is distributed under the terms of the Creative Commons Attribution-NonCommercial 4.0 International License (http://creativecommons.org/licenses/ by-nc/4.0/), which permits any noncommercial use, distribution, and reproduction in any medium, provided you give appropriate credit to the original author(s) and the source, provide a link to the Creative Commons license, and indicate if changes were made.

\section{REFERENCES}

1. Laumann AE. History and epidemiology of tattoos and piercings. Legislation in the United States. In: Dermatologic Complications with Body Art, 2009, p. 1-11.

2. Goldstein N. Tattoos defined. Clin Dermatol. 2007;25(4):417-20.

3. Hutton Carlsen K, Serup J. Patients with tattoo reactions have reduced quality of life and suffer from itch. Skin Res Technol. 2015;21(1):101-7.
4. Mataix J, Silvestre JF. Cutaneous adverse reactions to tattoos and piercings. Actas Dermo Sifiliográficas Engl Ed. 2009;100(8):643-56.

5. Jacob CI. Tattoo-associated dermatoses: a case report and review of the literature. Dermatol Surg. 2002;28(10):962-5.

6. Lubeck G, Epstein E. Complications of tattooing. Calif Med. 1952;76(2):83-5.

7. Cruz FAM, Lage D, Frigério RM, et al. Reactions to the different pigments in tattoos: a report of two cases. An Bras Dermatol. 2010;85(5):708-11.

8. Sowden JM, Byrne JP, Smith AG, et al. Red tattoo reactions: X-ray microanalysis and patch-test studies. Br J Dermatol. 1991;124(6):576-80.

9. Ashinoff R, Levine VJ, Soter NA. Allergic reactions to tattoo pigment after laser treatment. Dermatol Surg. 1995;21(4):291-4.

10. Clarke J, Black MM. Lichenoid tattoo reactions. Br J Dermatol. 1979;100(4):451-5.

11. Balfour E, Olhoffer I, Leffell D, et al. Massive pseudoepitheliomatous hyperplasia: an unusual reaction to a tattoo. Am J Dermatopathol. 2003;25(4):338-40.

12. Garcovich S, Carbone T, Avitabile S, et al. Lichenoid red tattoo reaction: histological and immunological perspectives. Eur J Dermatol EJD. 2012;22(1):93-6.

13. Corazza M, Zampino MR, Montanari A, et al. Lichenoid reaction from a permanent red tattoo: has nickel a possible aetiologic role? Contact Dermatitis. 2002;46(2):114-5.

14. Engel E, Santarelli F, Vasold R, et al. Modern tattoos cause high concentrations of hazardous pigments in skin. Contact Dermatitis. 2008;58(4):228-33.

15. Høgsberg T, Thomsen BM, Serup J. Histopathology and immune histochemistry of red tattoo reactions. Skin Res Technol ISBS Int Soc Digit Imaging Skin ISDIS Int Soc Skin Imaging ISSI. 2015;21(4):449-58.

16. Kaur RR, Kirby W, Maibach H. Cutaneous allergic reactions to tattoo ink. J Cosmet Dermatol. 2009;8(4):295-300.

17. Goldstein N. Mercury-cadmium sensitivity in tattoos. A photoallergic reaction in red pigment. Ann Intern Med. 1967;67(5):984-9.

18. Kazandjieva J, Tsankov N. Tattoos: dermatological complications. Clin Dermatol. 2007;25(4):375-82.

19. Hutton Carlsen K, Serup J. Photosensitivity and photodynamic events in black, red and blue tattoos 
are common: a 'Beach Study'. J Eur Acad Dermatol Venereol. 2014;28(2):231-7.

20. Serup J, Hutton Carlsen K. Patch test study of 90 patients with tattoo reactions: negative outcome of allergy patch test to baseline batteries and culprit inks suggests allergen(s) are generated in the skin through haptenization. Contact Dermatitis. 2014;71(5):255-63.

21. Antony FC, Harland CC. Red ink tattoo reactions: successful treatment with the Q-switched $532 \mathrm{~nm}$ Nd:YAG laser. Br J Dermatol. 2003;149(1):94-8.

22. Taaffe A, Knight AG, Marks R. Lichenoid tattoo hypersensitivity. Br Med J. 1978;1(6113):616-8.

23. Mortimer NJ, Chave TA, Johnston GA. Red tattoo reactions. Clin Exp Dermatol. 2003;28(5):508-10.

24. Winkelmann RK, Harris RB. Lichenoid delayed hypersensitivity reactions in tattoos. J Cutan Pathol. 1979;6(1):59-65.

25. Litak J, Ke MS, Gutierrez MA, et al. Generalized lichenoid reaction from tattoo. Dermatol Surg. 2007;33(6):736-40.

26. Sanghavi S, Dongre A, Khopkar U. Tattoo reactionsan epidemic on the surge: a report of 3 cases. Indian J Dermatol Venereol Leprol. 2013;79(2):231.

27. Campolmi P, Bassi A, Bonan P, et al. Cutaneous pseudolymphoma localized to black tattoo. J Am Acad Dermatol. 2011;65(5):e155-7.

28. Amann U, Luger TA, Metze D. Lichenoid pseudolymphomatous tattooing reaction. Hautarzt Z Für Dermatol Venerol Verwandte Geb. 1997;48(6):410-3.

29. Gardair Bouchy C, Kerdraon R, Kluger N, et al. Cutaneous lymphoid hyperplasia (pseudolymphoma) on the red dye of a tattoo. Ann Pathol. 2013;33(4):273-7.

30. Jaehn T, Kaiser A, Grüneis C, et al. Pseudolymphatic allergic immune reaction after tattooing. Organ Deutschsprachigen Arbeitsgemeinschaft Für Mikrochir Peripher Nerven Gefässe Organ Ver Dtsch Plast Chir. 2014;46(6):379-82.

31. Sweeney SA, Hicks LD, Ranallo N, et al. Perforating granulomatous dermatitis reaction to exogenous tattoo pigment: a case report and review of the literature. Am J Dermatopathol. 2013;35(7):754-6.

32. Godinho MM, Aguinaga F, Grynszpan R, et al. Granulomatous reaction to red tattoo pigment treated with allopurinol. J Cosmet Dermatol. $2015 ; 14(3): 241-5$.
33. Litvinov IV, Sasseville D. Pyoderma gangrenosum triggered by red tattoo dye. CMAJ Can Med Assoc J J Assoc Medicale Can. 2014;186(12):935.

34. Greve B, Chytry R, Raulin C. Contact dermatitis from red tattoo pigment (quinacridone) with secondary spread. Contact Dermatitis. 2003;49(5):265-6.

35. Collins P, Evans AT, Gray W, et al. Pulmonary sarcoidosis presenting as a granulomatous tattoo reaction. Br J Dermatol. 1994;130(5):658-62.

36. Nawras A, Alsolaiman MM, Mehboob S, et al. Case report: systemic sarcoidosis presenting as a granulomatous tattoo reaction secondary to interferon- $\alpha$ treatment for chronic hepatitis $\mathrm{C}$ and review of the literature. Dig Dis Sci. 2002;47(7):1627-31.

37. Antonovich DD, Callen JP. Development of sarcoidosis in cosmetic tattoos. Arch Dermatol. 2005;141(7):869-72.

38. Psaltis NM, Gardner RG, Denton WJ. Systemic sarcoidosis and red dye granulomatous tattoo inflammation after influenza vaccination: a case report and review of literature. Ocul Immunol Inflamm. 2014;22(4):314-21.

39. Sowden JM, Cartwright PH, Smith AG, Hiley C, Slater D. Sarcoidosis presenting with a granulomatous reaction confined to red tattoos. Clin Exp Dermatol. 1992;17(6):446-8.

40. Preda VA, Maley M, Sullivan JR. Mycobacterium chelonae infection in a tattoo site. Med J Aust. 2009;190(5):278-9.

41. Ravits HG. Allergic tattoo granuloma. Arch Dermatol. 1962;86(3):287-9.

42. Dang M, Hsu S, Bernstein E. Lichen planus or lichenoid tattoo reaction? Int J Dermatol. 1998;37(11):860-1.

43. Biro L, Klein W. Unusual complications of mercurial (cinnabar) tattoo: Generalized eczematous eruption following laceration of a tattoo. Arch Dermatol. 1967;96(2):165-7.

44. De Argila D, Chaves A, Moreno J. Erbium:Yag laser therapy of lichenoid red tattoo reaction. J Eur Acad Dermatol Venereol. 2004;18(3):332-3.

45. Kyanko ME, Pontasch MJ, Brodell RT. Red tattoo reactions: treatment with the carbon dioxide laser. J Dermatol Surg Oncol. 1989;15(6):652-6.

46. Madan V. Dermatological applications of carbon dioxide laser. J Cutan Aesthetic Surg. 2013;6(4):175-7. 
47. Zemtsov A, Wilson L. $\mathrm{CO}_{2}$ laser treatment causes local tattoo allergic reaction to become generalized. Acta Derm Venereol. 1997;77(6):497.

48. Green JB, Metelitsa AI. Optimizing outcomes of laser tattoo removal. Skin Ther Lett. 2011;16(10):1-3.

49. Kent KM, Graber EM. Laser tattoo removal: a review. Dermatol Surg. 2012;38(1):1-13.

50. Taylor CR, Gange R, Dover JS, et al. Treatment of tattoos by q-switched ruby laser: a dose-response study. Arch Dermatol. 1990;126(7):893-9.

51. Lehmann G, Pierchalla P. Tattooing dyes. Dermatosen Beruf Umw Occup Environ. 1988;36(5):152-6.

52. Vasold R, Naarmann N, Ulrich H, et al. Tattoo pigments are cleaved by laser light-the chemical analysis in vitro provide evidence for hazardous compounds. Photochem Photobiol. 2004;80(2): 185-90.
53. Saunders $\mathrm{H}, \mathrm{O}^{\prime}$ Brien $\mathrm{T}$, Nixon R. Textile dye allergic contact dermatitis following paraphenylenediamine sensitization from a temporary tattoo. Australas J Dermatol. 2004;45(4):229-31.

54. England RW, Vogel P, Hagan L. Immediate cutaneous hypersensitivity after treatment of tattoo with Nd:YAG laser: a case report and review of the literature. Ann Allergy Asthma Immunol Off Publ Am Coll Allergy Asthma Immunol. 2002;89(2):215-7.

55. Anderson RR, Geronemus R, Kilmer SL, et al. Cosmetic tattoo ink darkening. A complication of Q-switched and pulsed-laser treatment. Arch Dermatol. 1993;129(8):1010-4.

56. Herbich GJ. Ultrapulse carbon dioxide laser treatment of an iron oxide flesh-colored tattoo. Dermatol Surg. 1997;23(1):60-1. 